Journal for ImmunoTherapy of Cancer

\title{
Mesenchymal stem cells and oncolytic viruses: joining forces against cancer
}

\author{
Rafael Moreno ${ }^{1,2}$
}

To cite: Moreno $\mathrm{R}$.

Mesenchymal stem cells and oncolytic viruses: joining forces against cancer. Journal for ImmunoTherapy of Cancer 2021;9:e001684. doi:10.1136/ jitc-2020-001684

- Additional material is published online only. To view please visit the journal online (http://dx.doi.org/10.1136/jitc2020-001684).

Accepted 22 December 2020

Check for updates

(C) Author(s) (or their employer(s)) 2021. Re-use permitted under CC BY-NC. No commercial re-use. See rights and permissions. Published by BMJ.

${ }^{1}$ Virotherapy and immunotherapy group, ProCURE Program, Catalan Institute of Oncology - ICO, L'Hospitalet de Llobregat, Spain

${ }^{2}$ Cancer Virotherapy group, Oncobell Program, Institutd'Investigació Biomèdica de Bellvitge - IDIBELL,

L'Hospitalet de Llobregat, Spain

Correspondence to

Dr Rafael Moreno;

rafamoreno@iconcologia.net

\begin{abstract}
The development of oncolytic viruses (OVs) has increased significantly in the past 20 years, with many candidates entering clinical trials and three of them receiving approval for some indications. Recently, OVs have also gathered interest as candidates to use in combination with immunotherapies for cancer due to their immunogenic properties, which include immunogenic cell death and the possibility to carry therapeutic transgenes in their genomes. OVs transform non-immunogenic 'cold' tumors into inflamed immunogenic 'hot' tumors, where immunotherapies show the highest efficacy. However, in monotherapy or in combination with immunotherapy, OVs face numerous challenges that limit their successful application, in particular upon systemic administration, such as liver sequestration, neutralizing interactions in blood, physical barriers to infection, and fast clearance by the immune system. In this regard, the use of mesenchymal stem cells (MSCs) as cells carrier for OV delivery addresses many of these obstacles acting as virus carriers and factories, expressing additional transgenes, and modulating the immune system. Here, I review the current progress of OVs-loaded MSCs in cancer, focusing on their interaction with the immune system, and discuss new strategies to improve their therapeutic efficacy.
\end{abstract}

\section{BACKGROUND}

With more than 100 years of history, clinical experience using oncolytic viruses (OVs) for cancer treatment has alternated periods of highs and lows, showing a peak of interest during the 1950s and 1960s followed by years of abandonment due to poor clinical results and severe or even fatal adverse events observed after the first clinical trials (1970s and 1980s). ${ }^{1}$ It was not until the advent of molecular biology and gene manipulation techniques in the late 20th century that viruses regained interest as cancer treatments. These new techniques allowed scientists not only to isolate viruses with a natural selectivity for tumor cells, but also to modify existing viruses to target and replicate more selectively in cancer cells. Furthermore, an increase in the knowledge of viral biology allowed researchers to substitute viral sequences or to incorporate new DNA sequences (transgenes) into the viral genome, creating what is known as 'armed' OVs. These transgenes can exert diverse functions such as enhancing tumor tropism and penetration, increasing viral potency, promoting cell death, and antiangiogenesis, and modulating antitumor immunity, among others. ${ }^{2-4}$

To date, engineered OVs from more than 10 different viral families have been evaluated in phase I-III clinical trials, demonstrating favorable safety profiles. However, while local OVs administration has shown promising results in melanoma patients, systemic OVs administration has reported only occasional and transient responses. ${ }^{5}$ The inadequate delivery of OVs to the tumor, as a result of liver or spleen sequestration, immune system recognition and elimination, and poor tumor extravasation and spread, has been identified as a main factor for insufficient antitumor efficacy after systemic OVs administration. ${ }^{3} \mathrm{It}$ is, therefore, of clinical relevance to develop new strategies to improve OVs bioavailability and delivery to tumors, especially for those patients where intratumoral injection is not feasible or with tumor metastases.

This review will focus on one of these strategies: the use of mesenchymal stem cells (MSCs) as cell carriers for OVs and its effect on the immune system.

\section{MESENCHYMAL STEM CELLS}

As outlined above, OVs face numerous challenges that hinder their successful systemic application. For instance, once administered systemically, OVs can be filtered by and retained in some tissues, especially the liver. The immune system can also recognize these OVs in the bloodstream leading to their elimination. Moreover, in order to leave the bloodstream and enter the extracellular space, OVs have to overcome the abnormal tumor vasculature ${ }^{6}$ and the elevated interstitial fluid pressure. ${ }^{7}$ Finally, the tumor microenvironment (TME) bears several barriers that limit viral penetration and spreading such as an altered tumor extracellular matrix (ECM) rich in proteins, hyaluronic acid, proteoglycans, and stromal cells. ${ }^{8}$ Several 
A) Easy isolation by plastic adherence

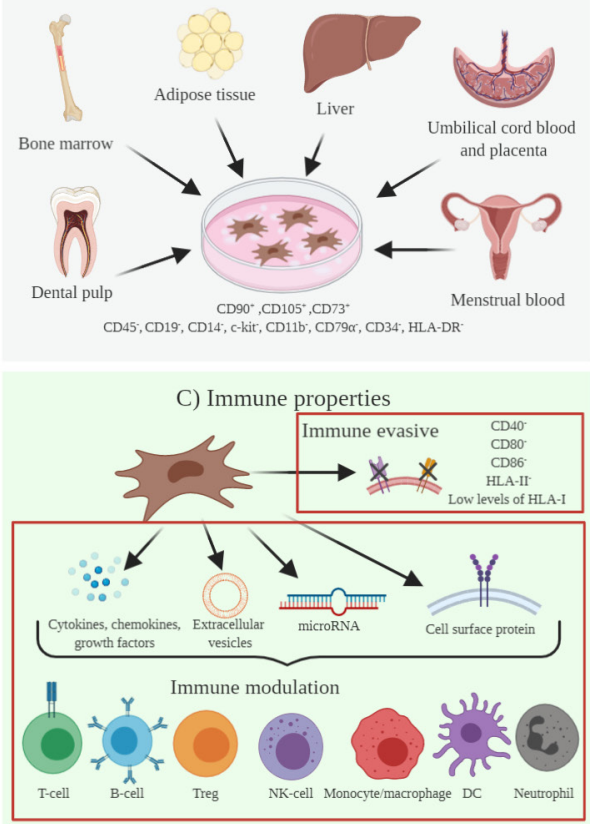

B) Self-renewing and multipotent differentiation capacity
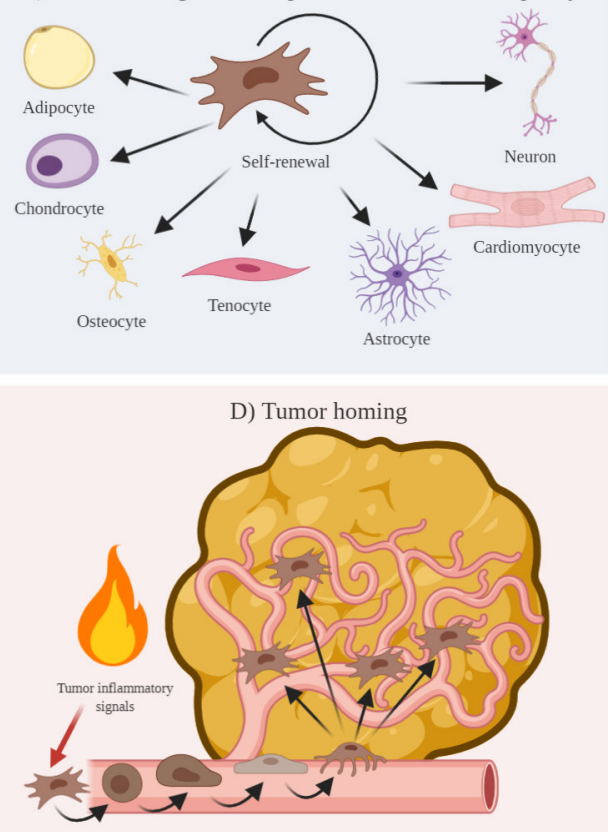

Figure 1 Mesenchymal stem cells (MSCs): origin, stem cell potential, and immunomodulatory properties. (A) MSCs can be easily isolated from diverse adult and fetal tissues, thanks to their adherence ability. Once isolated, and according to the

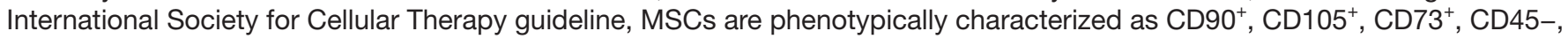
CD19-, CD14-, c-kit-, CD11b-, CD79 $\alpha-$, CD34-, and HLA-DR-. (B) MSCs are described as multipotent stem cells with the potential for self-renew and to differentiate into several cell types. (C) The lack of HLA-II and costimulatory molecules (CD40, CD80, and CD86) confers immune evasive properties to MSCs. Moreover, through the secretion of cytokines, chemokines, and growth factors, the production of extracellular vesicles, exosomes, and microRNAs, and the expression of cell surface proteins, MSCs exert immunomodulatory functions interfering with different pathways of the immune response. (D) In response to inflammatory signals expressed and secreted by tumor cells, MSCs have the potential to migrate to and to propagate within the tumor mass. DC, dendritic cell; HLA-DR, human leukocyte antigen-DR; NK: natural killer; Treg, regulatory T cells.

strategies are being developed in order to overcome these barriers (reviewed in ${ }^{910}$ ). Among them, the use of cell carriers for systemic delivery of OVs to the primary tumor and metastases represents an attractive system to address many of these obstacles. Thus, cell carries could protect OVs from complement or neutralizing antibodies, the two main viral clearance mechanisms in blood. Moreover, systemic administration of cells loaded with OVs could evade the filtering organs, and cross the endothelial barrier thanks to their capability to traffic towards different tissues and organs following chemokine gradients or using cell surface adhesion proteins. Finally, some types of cells have gained interest due to their ability to specifically migrate toward tumors such as immune cells ( $\mathrm{T}$ cells and macrophages), progenitor cells, cancer cells, and more recently neural stem cells and MSCs. ${ }^{11} 12$ From the plethora of cells used as cell carriers, MSCs are of particular interest because of their inherent properties, which will be discussed in the next section.

\section{MSCs: origin and properties}

In the 1970s, a novel subset of non-hematopoietic stem cells with a multilineage potential was described in the marrow stroma by Friedenstein and co-workers. ${ }^{13}$ They reported an adherent fibroblast-like cell with the ability to generate colonies in vitro (figure 1). These cells, defined as colony-forming unit fibroblasts, demonstrated later the ability to differentiate both in vitro and in vivo into a variety of cell types of mesenchymal origin such as adipocytes, chondrocytes, osteocytes, and tenocytes, and into other cell types such as cardiomyocytes, myocytes, astrocytes, and neurons, among others. ${ }^{14}$ It was at the end of the 1980s when further studies led to characterize the heterogeneity of the marrow stromal cell pool and to identify a stem cell population with a mesodermal germ layer origin that contributed to the formation of connective tissue, skeletal muscle cells, and vascular system. These cells are known as marrow stroma stem cells or bone marrow-derived MSCs (BM-MSCs). ${ }^{15} 16$ Of special interest were the experiments performed by several groups showing that BM-MSCs had unique immunomodulatory properties in an allogeneic transplant. ${ }^{17}$

During the following decades, and thanks to their adherence properties and to new MSCs isolation procedures, an increasing number of human MSCs were identified from diverse organs and tissues such as adipose tissue, muscle, liver, periosteum, dental pulp, perichondrium, menstrual blood, etc. ${ }^{18}$ MSCs were also described in fetal fluids and tissues, including amniotic fluid, cord blood, placenta, bone marrow, blood, liver, and lung. ${ }^{19} 20$ Interestingly, although both fetal and adult MSCs share 
immunological attributes, ${ }^{21}$ those from fetal origin are present in higher amounts and have shorter cell doubling time and higher proliferative capacity than those from adult tissues. ${ }^{22}$

To date, there are no unique markers to unequivocally identify MSCs and the presence or the absence of a set of non-specific markers are required to phenotypically characterize them. This challenging phenotypic marker landscape has prevented the proper identification of MSCs isolated from different laboratories. ${ }^{23}$ In 2006, the International Society for Cellular Therapy defined MSCs as self-renewing multipotent fibroblast-like cells with plastic adherent capacity, capable to differentiate, at least, into adipocytes, osteocytes, and chondrocytes, positive for expression of CD90, CD105, and CD73, and negative for CD45, CD19, CD14, c-kit, CD11b, CD79 $\alpha$, CD34 (except adipose tissue-derived MSCs that express CD34 in short-term cultures), and human leukocyte antigen-DR (HLA-DR). ${ }^{24}$

MSCs present unique immunological and immunomodulatory functions. Thus, MSCs are considered to have low immunogenicity owing to the lack of expression of HLA class II, CD40, CD80, and CD86 co-stimulatory molecules, and low levels of expression of HLA class I on their surface. ${ }^{25}$ Although it has been widely considered that this immunological privileged phenotype allows MSCs to evade immune system recognition, recent reports have shown that MSCs can elicit both humoral and cellular immune responses, thus suggesting an immune evasive rather than an immune privileged immunophenotype. ${ }^{26}$

MSCs also exert immunomodulatory functions over almost all immune cells through cell-to-cell contact and by secreting a wide variety of cytokines, chemokines, and growth factors. Thus, MSCs have been described to regulate the activity of naive, memory, and effector T cells, B cells, regulatory T cells (Tregs), natural killer cells (NKs), dendritic cells, monocytes/macrophages, and neutrophils. ${ }^{27} 28$

Finally, and of special interest for systemic cancer treatment, MSCs are known to migrate to sites of injury and inflammation, which are two traits of the TME. ${ }^{29}$ MSCs respond to inflammation mediators that are highly expressed in the tumor such as growth factors (vascular endothelial growth factor (VEGF), fibroblast growth factor (FGF), granulocyte-macrophage colonystimulating factor, hematopoietic growth factor, hepatocyte growth factor (HGF), epidermal growth factor (EGF), transforming growth factor beta-1 (TGF- 31 ), granulocyte colony-stimulating factor or stromal-derived growth factor-1 $\alpha$ ), chemokines (CCL2, CCL3, and CXCL12), cytokines (interleukin 6 (IL-6) and IL-8), integrins (vascular cell adhesion molecule 1 (VCAM-1) and intracellular adhesion molecule-1 (ICAM-1)), and selectins (P-selectin and E-selectin). ${ }^{30}$ In response to these local tumor inflammatory mediators, MSCs initiate a rolling process similar to that described for leukocytes in response also to inflammation, whereby MSCs adhere to the vascular epithelium and begin transmigration into the tumor. ${ }^{31}$
Evidence of MSCs tumor migration has been reported for several cancer types, pointing out their potential as delivery vectors for antitumor therapies. ${ }^{32}$

Their easy isolation and expansion, in addition to their immune evading properties and their ability to migrate to the tumor, make MSCs particularly appealing as cell carriers for OVs.

\section{MSCs and cancer}

The effect of MSCs on tumor progression has been a controversial field for the last decades, with several studies suggesting a role of MSCs in promoting tumor growth and metastasis, while others support their antitumor potential. ${ }^{33}$ In recent years, the pro-tumorigenic theory has gained force based on studies that demonstrate the effect of MSCs in the TME and its regulation in tumor immunosurveillance.

\section{Modulation of the TME by MSCs}

To better understand the effect of MSCs on the TME, it is important to note that tumors have long been considered as a wound process that fails to resolve. ${ }^{34} 35$ Thus, it has been described how wound healing and tumor progression share common traits like inflammation, tissue proliferation and remodeling, ECM remodeling, immune cell mobilization and activation, and neovascularization. However, while this is a well-defined process for wound healing, in cancer this process is not properly completed and becomes an unresolved situation.

MSCs contribution to tumor inflammation, tissue proliferation and remodeling steps have been extensively reviewed by Li et al, ${ }^{36}$ who highlight the key role of tumorassociated MSCs (TA-MSCs) at the first stages of cancer development. TA-MSCs in the tumor inflammatory microenvironment secrete cytokines and chemokines such as IL-6, HGF, CCL-2, CXCL1/2, HGF, or CXCL3, that promote infiltration of myeloid-derived suppressor cells (MDSCs) and monocytes, and differentiation of the latter to M2 macrophages. Additionally, TA-MSCs participate in recruiting new MSCs to tumors where they support tumor proliferation through their transdifferentiation into tumor cancer-associated fibroblasts, which in turn provide survival and proliferative signals to cancer stem cells by secreting growth factors and cytokines such as HGF, TGF- $\beta$, FGF, IL-6, IL- 8 , CXCL1/ 7 , and bone morphogenetic protein. TA-MSCs and recruited MSCs also secrete VEGF and platelet-derived growth factor, which support the neovascularization of the growing tumor. In the last step of tumor progression, the remodeling step, MSCs role is contradictory. On one hand, TA-MSCs release exosomes containing microRNAs, which reduce the tumor growth rate by promoting a dormancy phenotype in tumor cells, and also inhibit angiogenesis. These antitumoral functions are considered a reminiscence of their anti-inflammatory cellular program. On the other hand, TA-MSCs secrete chemokines (CCL-2, CCL-5, CCL9, and CXCL10) and cytokines (IL-8, IL-1 $\beta$, tumor necrosis factor-alpha $(\mathrm{TNF} \alpha)$, and $\mathrm{TNF} \beta)$ that stimulate 
the epithelial-mesenchymal transition, increasing tumor cell invasion and metastasis.

\section{Regulation of tumor surveillance by MSCs}

Cytokines and exosomes secreted by TA-MSCs, as a result of their crosstalk with tumor cells, modulate the tumor surveillance acting on different players of the innate and the adaptive immune system. ${ }^{37}$ Regarding the innate immune system, TA-MSCs stimulate recruitment and differentiation of immunosuppressive macrophages M2, via secretion of prostaglandin E2 (PGE2) and exosomes, ${ }^{38} 39$ and of MDSCs, through the secretion of HGF and CXCL3. ${ }^{40}$ Moreover, IL-6, PGE2, and microRNAs secreted by MSCs also prevent differentiation of monocytes to dendritic cells (DCs), and maturation of immature into mature DCs. ${ }^{41}$ TGF- $\beta$, IL-6, PGE2, and microRNAs secreted by TA-MSCs are responsible for the impaired function of NK cells, in some cases downregulating the expression of activating NK cell receptors: NKp44, NKp30, NKG2D, DNAX accessory molecule-1 (DNAM-I), and NKG2A. ${ }^{42}{ }^{43}$ Finally, MSCs exert a potent $\gamma \delta$-T cell immunosuppression through cyclooxygenase-2 (COX2)-dependent production of PGE2. ${ }^{44}$

MSCs also immunosuppress the adaptive immunity in the tumor. MSCs can inhibit naïve and memory T cell responses by upregulating lymphocyte functionassociated 3, ICAM-1, and VCAM-1. ${ }^{45}$ Additionally, TA-MSCs impair correct effector lymphocyte activation by secreting inhibiting factors such as TGF- $\beta$, PGE2, IL-10, indoleamine 2,3-dioxygenase and nitric oxide. ${ }^{45}$ Furthermore, TA-MSCs exert a potent TME immunosuppressive effect by increasing the amount of Tregs in the tumor. ${ }^{47}$

Altogether, these MSC functions in the TME support the role of MSCs in tumor progression and spread. Therefore, it is necessary to carefully modify MSCs prior to their application as antitumor therapies, by knocking down some of these pro-tumoral properties, inserting suicide gene as 'safety switch', or combining with OVs that eventually eliminate infected MSC during the treatment. In line with this, modified MSCs have been evaluated in several animal models, ${ }^{30}$ and their safety and efficacy have been reported in early clinical trials for cancer treatment. ${ }^{48}$ No malignant or pro-tumoral effects have been reported from any of these studies, indicating that modified MSCs lack the tumor-promoting properties described above.

\section{APPLICATION OF MSCS AS CELL CARRIER FOR SYSTEMIC DELIVERY OF OVS}

To serve as efficient cell carriers for OVs delivery to tumors on systemic administration, MSCs should fulfill the following requirements: (1) efficient infectivity, (2) allow OV replication and production, (3) modulate the immune response against the virus, and (4) promote an antitumor immune response (figure 2).

Although multiple types of viruses can be combined with MSCs, there are different factors that should be considered to maximize the benefit of the combination.

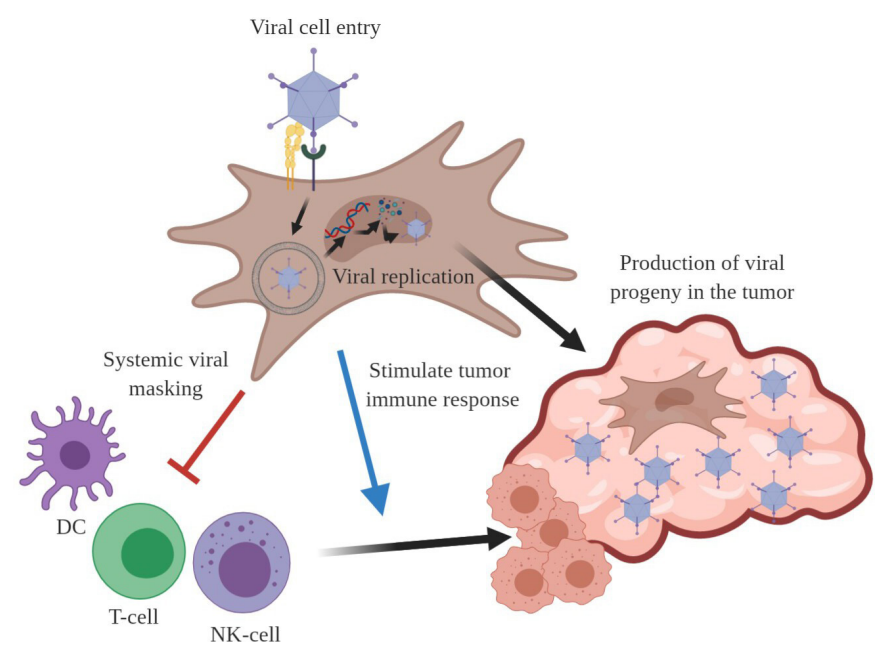

Figure 2 To be considered as efficient cell carriers for OVs, MSCs have to be permissive to infection and replication of the OV of choice. Moreover, on systemic administration, MSCs must protect the OV from immune system recognition in order to have successful tumor delivery. Once in the tumor, MSCs should spread to favor a homogeneous production of new viral particles within the tumor mass. Finally, a desirable property for ideal OV cell carriers is the capacity to stimulate or promote tumor immune system recognition and eventually antitumor immune response. DC, dendritic cell; MSCs, mesenchymal stem cells; NK, natural killer; OV, oncolytic virus.

Thus, viral genome type is an important consideration since RNA viruses replicate faster than DNA viruses, allowing less time for MSCs to reach the tumor. Choosing OVs capable of infecting and replicating properly in MSCs is needed in order to ensure an optimal tumor viral delivery. Delivery via MSCs is specifically suitable for those viruses lacking tumor tropism, since they could benefit from MSCs tumor homing properties, not only in terms of tumor delivery but also to avoid normal organ infection. Finally, the use of MSCs as cell vehicles represents a particular advantage for viruses commonly infecting humans where pre-immunity in the population is broadly present, such as measles virus (MV) or adenovirus (Ad).

Several preclinical studies have evaluated the combination of human MSCs and OVs in the setting of systemic administration (online supplemental table 1).

\section{Myxoma virus (MYXV)}

MYXV expressing green fluorescent protein (GFP) as reporter gene has been combined with human adiposederived MSCs (ASCs) to treat malignant brain tumor in mice. ${ }^{49}$ This study demonstrated not only the capability of ASCs to generate new viral particles, but also the tumor-homing properties of infected cells on intracranial administration. As a result, a significant increase in survival was observed in mice treated with the OV-loaded MSCs in comparison with mice treated with MSCs alone.

BM-MSCs have also shown to be permissive to MYXV replication. ${ }^{50}$ Moreover, using an IL-15-armed MYXV to infect BM-MSCs, the authors demonstrated a superior 
antitumor efficacy in an immune competent pulmonary melanoma model after intravenous treatment, compared with MYXV monotherapy. An elevated percentage of circulating NK cells was observed only in those animals treated with the virus without cell carriers, indicating that MSCs potentially prevented recognition of the virus by the immune system. Finally, an increase of pro-inflammatory cytokines (interferon (IFN)- $\gamma$ and TNF $\alpha$ ), programmed cell death protein 1 (PD-1)/programmed death-ligand 1 (PD-L1), and effector T cells infiltration was documented only in tumors from animals treated with MYXV-IL-15loaded MSCs, indicating a possible antitumor immune response as a consequence of the therapy.

\section{Herpes simplex virus (HSV)}

Two different studies have demonstrated the antitumor potential of the combination of HSV and MSCs. Leoni and co-workers infected fetal membrane-derived MSCs (FM-MSCs) with an HSV encoding for a single-chain antibody targeting human epidermal growth factor receptor 2 (HER-2) (R-LM249). ${ }^{51}$ FM-MSCs viral infection, viral progeny production, and tumor viral delivery were demonstrated. The antitumor efficacy on intravenous administration was evaluated in two different mice models. In a lung metastases model (using the HER-2 positive SK-OV-3 cell line), R-LM249-infected MSCs significantly inhibited the appearance of metastasis, unlike uninfected MSCs. Similar results were observed in a model of brain metastasis (HER2 ${ }^{+}$MDA-MB-453 EGFP cell line), in which R-LM249-infected FM-MSCs prevented the development of brain metastases. In a second study, Du et al reported MSC-mediated delivery of oncolytic-HSV to brain metastases in a murine model of melanoma after a intracarotid single administration. ${ }^{52}$ This tumor delivery resulted in a significant remission of metastatic tumors and mice survival in comparison to MSCs-treated alone.

\section{Measles virus (MV)}

MSCs from diverse origins have been combined with MV for cancer treatment. Two groups have evaluated intravenous single administration of MV-loaded BM-MSCs in hepatocellular carcinoma and lymphoblastic leukemia mouse models. ${ }^{53}{ }^{54}$ Both studies reported BM-MSCs permissiveness for virus replication and, most importantly, virus delivery to tumors and higher therapeutic efficacy than cell-free MV in the presence of pre-existing neutralizing anti-MV antibodies. MSCs from adipose tissue have also been combined with MV. Mader et al tested MV-infected ASCs antitumor potential in a xenograft murine model of ovarian cancer after a single intraperitoneal administration. ${ }^{55}$ In line with the studies described above, the use of ASCs as cell carriers not only enhanced the therapeutic potential in comparison with virus alone treatment, but also the capacity to protect the virus from pre-existing antiviral immunity. In a follow-up study, the same authors pointed out the feasibility of using patientderived MSC as carriers for $\mathrm{MV}^{56}$
Importantly, these three studies show for the first time the advantage of using MSCs as cell carriers when viral pre-immunity exists.

\section{Adenovirus (Ad)}

Oncolytic Ads (OAds) have been the most frequently used viruses in combination with MSCs for cancer treatment. Pioneer studies in 2007 compared Ad infectivity and viral production in MSCs using chimeric or modified adenoviral fibers. ${ }^{57} 58$ These studies also highlighted the capacity of OAd-loaded MSCs to reach the tumor after single intravenous administration, and to reduce tumor growth in lung and breast tumor murine models.

The group of Frederick Lang evaluated the combination of BM-MSCs with the OAd delta-24/RGD. In a first work using two different glioma xenograft murine models (U87 and U215-V121), the authors detected brain tumor tropism and antitumor activity of infected MSCs after single intracarotid administration. ${ }^{59}$ A second study demonstrated a main role of TGF- $\beta$ secreted by glioma stem cells in the BM-MSCs glioma tropism. ${ }^{60}$

Bolontrade et al described a subpopulation of BM-MSCs, overexpressing integrins $\alpha 2, \alpha 3$, and $\alpha 5$, with and enhanced tumor migration capacity ${ }^{61}$ Loading these cells with an OAd led to a significant therapeutic efficacy compared with unloaded MSCs and virus as monotherapies in a melanoma xenograft model after a single retro orbital administration.

Hypoxic conditions have been also reported to favor MSCs tumor-homing properties by inducing the upregulation of CXCR4 and CXCR1 receptors. ${ }^{62}$ However, the combination of an E1B-55KD-deleted OAd with BM-MSCs cultured under hypoxic conditions did not control tumor growth after a single intraperitoneal administration in a xenograft model of colon cancer. Authors attributed the low potency of the virus as a possible explanation for this low efficacy, although no control group with virus as monotherapy was included in the study. The combination with a more potent OAd would have been desirable to demonstrate the benefit of culturing BM-MSCs in hypoxia for this type of cancer therapy.

Hepatocellular carcinoma murine models have been used to evaluate the efficacy of umbilical cord-derived MSCs (HU-MSCs) and BM-MSCs loaded with different OAds. Combining an OAd expressing IL-24 with HU-MSCs engineered to express the E1A adenoviral protein, $\mathrm{Li}$ and colleagues reported successful virus replication and virus production, and efficient migration of virus-loaded HU-MSCs to tumors after a single intravenous administration. ${ }^{63}$ Furthermore, addition of a low-dose 5-fluorouracil to the OAdv-infected HU-MSCs combination resulted in effective tumor growth inhibition. HU-MSCs were also combined with an OAd expressing a microRNA-122 target sequence to avoid virus replication in normal liver cells. ${ }^{64}$ Significant antitumor efficacy was reported after two intravenous administrations using orthotopic and subcutaneous hepatocarcinoma tumor models, although direct comparison with virus alone was not performed. Finally, 
BM-MSCs as cell carriers for an OAd encoding a WNTinhibiting (WNTi) decoy receptor have been tested in an orthotopic hepatocellular carcinoma. After optimizing the OAd dose for loading into BM-MSCS, two intravenous administrations of OAd-WNTi-loaded BM-MSC showed remarkable therapeutic efficacy in vivo ${ }^{65}$. Interestingly, direct comparison with OAd-WNTi without cell carriers showed 8.1-fold greater therapeutic efficacy for cellprotected viruses compared with the 'naked' virus group. Moreover, MSCs efficiently extended OAd blood circulation time while preventing hepatic damage in comparison with direct virus administration. In a follow-up study, the same group used a dynamic in silico TME model interaction network and a predictive mathematical model to identify two parameters to improve the outcome: the multiplicity of infection used to infect MSCs and the number of OAd-MSCs administered. ${ }^{66}$

Ad/RLX, an OAd coding for relaxin, a peptide hormone with ECM collagen degradation properties, has been combined with BM-MSCs for preclinical pancreatic cancer treatment. ${ }^{67}$ Nude mice carrying AsPC-1 pancreatic xenograft tumors received three intravenously administration of naked Ad/RLX, Ad/RLX-loaded BM-MSCs or BM-MSCs infected with Ad/RLX previously treated with poly(ethyleneimine)-conjugated poly(CBA-DAH) (PCDP), a cationic polymer that increases MSCs viral infectivity. Significant antitumor efficacy was observed only for the last group, probably due to the higher MSCs infectivity and viral production of PCDP-treated Ad/RLX.

Using xenograft chick embryo model of pancreatic cancer, Kaczorowski et al showed the capacity of OAdinfected BM-MSCs to invade tumor spheroids generating new viral particles in the tumor. ${ }^{68}$ Moreover, using a chimeric OAd (fiber 5/3) expressing TNF-related apoptosis-inducing ligand as load for BM-MSCs, increased tumor reduction was determined compared with the virus monotherapy.

MSCs isolated from menstrual blood (MenSCs) have been also used as cell carriers for OAd. Guo et al used MenSCs for tumor delivery of a chimeric OAd in murine models of colorectal cancer. ${ }^{69}$ After intravenous or intraperitoneal administration, correct MSCs tumor migration was determined. Moreover, two intravenous administrations of Ad5/F11-infected MenSCs resulted in significant antitumor efficacy although viral treatment alone was not included in the study as a control.

ICOVIR15, an E1a/delta24-modified promoter and RGD fiber OAd, has been used in combination with MSCs for cancer treatment. The group of M Brenner used BM-MSCs infected with an ICOVIR15 engineered to express an inducible Caspase 9 suicide gene (ICOVIR15-Ad.iC91) for preclinical lung cancer treatment. ${ }^{70}$ After one single intravenous administration, MSCs could be detected in tumor tissues and higher tumor control was observed in animals treated with ICOVIR15-Ad.iC9-MSCs.

We have focused on using MenSCs as an alternative ICOVIR15 cell carrier. In a first study, viral capsid modifications were compared to select the most efficient in terms of MSCs infection and viral progeny production. ${ }^{71}$ Using a lung xenograft murine model, tumor homing potential of uninfected and infected MenSCs was demonstrated after intraperitoneal administration. A subsequent study reported the benefit of using allogeneic instead of autologous MenSCs, as a consequence of the higher pro-inflammatory environment generated when OAd-loaded MenSCs interacted with allogeneic peripheral blood mononuclear cells (PBMCs). ${ }^{72}$ Activation of both $\mathrm{T}$ cells and NK cells was associated with this pro-inflammatory MSCs phenotype indicating the potential capability of Ad-infected MSCs to favor tumor immune recognition. The therapeutic potential of OAdMenSCs was demonstrated in tumor-bearing NSG mice who received intravenous injections of allogeneic human PBMCs. Monocytes and NK cells contributed to efficacy. Finally, the therapeutic potential of OAd-MenSCs has been further improved by loading MenSCs with an ICOVIR15 oncolytic vector engineered to express an EGF receptor-targeting bispecific T cell engager (cBiTE). Antitumor efficacy of ICOVIR15-cBiTE MenSCs was superior to both monotherapy controls. Altogether, these results suggest that to improve viral-loaded MSCs therapy OAd should be optimized. ${ }^{73}$

Finally, two different groups have demonstrated the preclinical benefit of using OAd-loaded MSCs to treat brain tumors after local or intratumoral administration. Sonabend and co-workers reported that injecting OAdMSCs $5 \mathrm{~mm}$ away from brain lesions, promote cell migration to the tumor which results in local virus production. No efficacy experiment was performed in this study. More recently, Martinez-Quintanilla et al employed ICOVIR17, an ICOVIR15 modified to encode for soluble hyaluronidase PH20 that mediates ECM hyaluronic acid degradation, in combination with ASCs to treat glioblastoma (GBM) intratumorally. ${ }^{74}$ A significant antitumor efficacy was observed in mice bearing intracranial U87 tumors or patient-derived GBM xenografts.

In summary, MSCs from diverse origins have demonstrated potential as cell carriers for RNA or DNA OVs with different degrees of lytic properties (ie, low or high), ${ }^{75}$ highlighting the versatility of these cells to combine with OVs.

\section{CLINICAL EXPERIENCE USING MSCS AS CELL CARRIER FOR OVS}

According to the clinicaltrials.org database, there are six registered clinical trials (completed or recruiting) evaluating the safety and therapeutic efficacy of OVs using MSCs as cell carriers for cancer treatment (online supplemental table 2).

BM-MSCs carrying ICOVIR-5, an OAd, ${ }^{76}$ have been evaluated in four cancer clinical trials. The first trial assessed the use of CELYVIR (X-ray irradiated ICOVIR-5-loaded autologous BM-MSCs) to treat pediatric refractory neuroblastoma. Clinical results from the first four patients 
treated (refractory stage IV neuroblastoma, pre-treated with at least two front-line multichemotherapy before CELYVIR) revealed the absence of toxicity or disease progression related to the infusion of CELYVIR. ${ }^{77}$ The first enrolled patient experienced a partial remission after the third CELYVIR infusion which turned into a complete response after standard consolidation with high-dose chemotherapy, highlighting the therapeutic potential of the therapy. An immune response secondary to the inflammatory oncolysis produced by ICOVIR-5 was suggested as a major therapeutic mechanism. Clinical outcome from additional 12 patients treated with CELYVIR showed eight progressive diseases (PDs), one stable disease (SD), three partial responses (PR), and one complete response (CR) ${ }^{78}$ Secondary peaks of viral replication were detected in 7 out of 12 patients 7 days after CELYVIR infusions, without differences between responder $(\mathrm{SD}+\mathrm{PR}+\mathrm{CR})$ and non-responder (PD). Biomarker analysis revealed suggestive factors associated to response, including MSCs high expression levels of two chemokine receptors (CCR1 and CXCR1, both directly involved in MSCs tumor homing ability), higher number of circulating $\mathrm{T}$ cells at baseline, and a less pro-inflammatory MSC profile after adenoviral infection. It is important to note that no correlative analysis and clinical outcome were performed due to small patient population.

A second completed study using CELYVIR for systemic treatment of adult and pediatric advance solid tumors has been reported (NCT01844661). ${ }^{79}$ Nine pediatric and seven adult patients completed the therapy (CELYVIR once weekly for six cycles). No grade 2-5 toxicities related to CELYVIR were reported confirming the treatment safety. Second wave of Ad replication was detected in seven out of nine pediatric patients but not in adult patients. Regarding antitumor efficacy, two pediatric patients (neuroblastoma) and one adult patient (anaplastic astrocytoma) showed stabilization of the disease. Because of the small sample and limited clinical outcome, no conclusion could be drawn from this trial, and a phase II trial has been proposed to further evaluate the therapy benefit.

Although the presence of infected BM-MSCs in the tumor has not been measured in these complete trials, the clinical outcome does not seem to be directly related with the CELYVIR doses received. Assuming a dosedependent homing of MSC to tumors, this lack of dosedependent clinical response points to other factors rather than the amount of modified MSCs in the tumor that could contribute to the efficacy.

Two additional trials using ICOVIR5 are ongoing, in which allogeneic, instead of autologous, BM-MSCs are used as carriers (defined as aloCELYVIR). In the first one, aloCELYVIR is combined with chemotherapy and radiotherapy for the treatment of children and adolescents with relapsed or refractory extracranial solid tumors (EudraCT number: 2019-001154-26). In the second one, aloCELYVIR is used to treat patients with metastatic uveal melanoma (EudraCT number: 2020-005207-39). No results have been reported thus far.
Combination of allogeneic BM-MSCs and the OAd DNX-2401 (a delta24-RGD Ad) is being evaluated in patients with recurrent GBM, gliosarcoma, or wild-type IDH-1 anaplastic astrocytoma in a phase I clinical trial (NCT03896568). No results have been disclosed yet.

Finally, phase I/II clinical trial is ongoing evaluating the use of ASCs as cell carriers for MV-NIS, an MV encoding thyroidal sodium iodide symporter (NCT02068794). The primary objectives of this trial are to determine the maximally tolerated dose of ASC-loaded MV-NIS intraperitoneally administered (phase I), and to assess the 4-month progression free survival of ovarian cancer patients treated with this therapy in a phase II expansion cohort. Interestingly, one of the tertiary objectives of the trial is to assess biodistribution of virally infected cells using singlephoton emission CT/CT imaging, which could show for the first time the MSCs tumor tropism in patients, validating the use of radionuclide reporter genes to track OV-loaded MSCs kinetics in clinical trials. The interim analysis for this study is expected in early 2021.

\section{CONCLUSIONS AND FUTURE DIRECTIONS}

Although the number of preclinical studies evaluating the use of MSCs as cell carriers for OVs is limited, this strategy has demonstrated superior to direct OV administration in those studies where both therapies were directly compared. Additionally, data from clinical studies using OV-loaded MSCs have shown systemic protection of the virus from neutralizing immune responses and clinical benefit, including a complete response. Of note, this clinical efficacy has been obtained using 'non-armed' OVs designed more than a decade ago. Therefore, a logical next step aiming at improving clinical outcome should focus on combining MSCs with more potent OVs. The number of armed and genetically modified OVs under clinical investigation to date is extensive, ${ }^{3}$ which opens a wide variety of opportunities for combinations with MSCs.

It is also possible to improve the clinical outcome by optimizing the MSCs. Although bone marrow represents the main and most frequent source for MSCs isolation and amplification, new sources are emerging as excellent candidates for MSC isolation because are less invasive or render MSCs with higher amplification potential, such as adipose tissue and menstrual blood. MSCs subpopulations enriched in integrins ${ }^{61}$ or growing MSCs under hypoxic conditions ${ }^{62}$ could be considered excellent approaches to enhance MSCs tumor tropism. In this scenario, it is even more interesting to have an abundant source for MSCs that can be enriched in the subpopulation of interest, such as menstrual blood with millions of potential monthly donors. Infectivity and viral production could be also optimized using polymers or different viral capsids. ${ }^{6771}$ Finally, the protumoral phenotype of MSCs could be converted to an antitumoral one under certain conditions according to the new paradigm for MSCs described in 2010 by the group of Betancourt. ${ }^{80} 81$ This group described how using diverse Toll-like receptor (TLR) agonists, MSCs could be 
polarized into a pro-inflammatory MSC1 or an immunosuppressive MSC2 phenotype, with MSC1 showing antitumor potential, whereas MSC2 promoting tumor growth and metastasis. ${ }^{82}$ This theory was demonstrated for TLR4-primed MSCs (polarized toward a MSC1-tumor growth inhibition phenotype) and TLR3-primed MSCs (polarized toward a MSC2 immunosuppressive phenotype).$^{83}$ Recently, we also showed how infection by an OAds induces TLR9 overexpression and activation of the NF-אB pathway in menstrual blood-derived MSCs leading to a specific cytokine secretion profile and $\mathrm{T}$ cells and NK cells activation. ${ }^{72}$ Therefore, blocking or activating specific MSCs TLRs might be an effective strategy to add MSCs immune-stimulatory properties to OV oncolysis in order to enhance the therapy antitumor potential.

In conclusion, although results from ongoing clinical trials will better define the clinical potential of MSCs as cell carriers of OVs, new efforts should be made in order to translate recent viral and cellular preclinical improvements to the clinic, either in monotherapy or in combination with radiotherapy, chemotherapy or even immunotherapies.

Correction notice This paper has been updated since first published to add reference 49 .

Acknowledgements The author acknowledges Ramon Alemany and Carlos A Fajardo for extensive revision of this manuscript. Figures were created with BioRender.com. We thank CERCA Program/Generalitat de Catalunya for their institutional support.

\section{Collaborators Ramon Alemany.}

Contributors RM: literature search and review, writing, graphical design, and editing.

Funding This work was supported by the Asociación Española Contra el Cáncer, BI02017-897554-C2-1-R from the Ministerio de Economía y Competitividad of Spain, Adenonet BI02015-68990-REDT from the Ministerio de Economía y Competitividad of Spain, Red ADVANCE(CAT) project COMRDI15-1-0013 from Ris3CAT and 2017SGR449 research grant from the 'Generalitat de Catalunya', and was cofunded by the European Regional Development Fund, a way to Build Europe.

Competing interests None declared.

Patient consent for publication Not required.

Provenance and peer review Not commissioned; externally peer reviewed.

Open access This is an open access article distributed in accordance with the Creative Commons Attribution Non Commercial (CC BY-NC 4.0) license, which permits others to distribute, remix, adapt, build upon this work non-commercially, and license their derivative works on different terms, provided the original work is properly cited, appropriate credit is given, any changes made indicated, and the use is non-commercial. See http://creativecommons.org/licenses/by-nc/4.0/.

\section{REFERENCES}

1 Kelly E, Russell SJ. History of oncolytic viruses: genesis to genetic engineering. Mol Ther 2007;15:651-9.

2 Hemminki O, dos SJM, Hemminki A. Oncolytic viruses for cancer immunotherapy. BioMed Central 2020;13:1-15.

3 Zheng M, Huang J, Tong A, et al. Oncolytic viruses for cancer therapy: barriers and recent advances. Mol Ther Oncolytics 2019;15:234-47.

4 Chaurasiya S, Fong Y, Warner SG. Optimizing oncolytic viral design to enhance antitumor efficacy: progress and challenges. Cancers 2020;12:1699.

5 Li Z, Jiang Z, Zhang Y, et al. Efficacy and safety of oncolytic viruses in randomized controlled trials: a systematic review and metaanalysis. Cancers 2020;12:1416.
6 Mizukami Y, Sasajima J, Ashida T, et al. Abnormal tumor vasculatures and bone marrow-derived pro-angiogenic cells in cancer. Int $J$ Hematol 2012;95:125-30.

7 Baronzio G, Parmar G, Baronzio M. Tumor Interstitial Fluid: Proteomic Determination as a Possible Source of Biomarkers. Cancer Genomics - Proteomics. International Institute of Anticancer Research 2014;11:225-37.

8 Harisi R, Jeney A. Extracellular matrix as target for antitumor therapy. Onco Targets Ther 2015;8:1387.

9 Hill C, Carlisle R. Achieving systemic delivery of oncolytic viruses. Expert Opin Drug Deliv 2019;16:607-20.

10 Harrington K, Freeman DJ, Kelly B, et al. Optimizing oncolytic virotherapy in cancer treatment. Nat Rev Drug Discov 2019;18:689-706.

11 Willmon C, Harrington K, Kottke T, et al. Cell carriers for oncolytic viruses: fed ex for cancer therapy. Mol Ther 2009;17:1667-76.

12 Nakashima H, Kaur B, Chiocca EA. Directing systemic oncolytic viral delivery to tumors via carrier cells. Cytokine Growth Factor Rev 2010;21:119-26.

13 Friedenstein AJ, Petrakova KV, Kurolesova Al, et al. Heterotopic transplants of bone marrow. Transplantation 1968;6:230-47.

14 Han Y, Li X, Zhang Y, et al. Mesenchymal stem cells for regenerative medicine. Cells 2019;8:886.

15 Caplan Al. The mesengenic process. Clin Plast Surg 1994;21:429-35.

16 Owen M. Marrow stromal stem cells. J Cell Sci Suppl 1988;10:63-76.

17 Patel SA, Sherman L, Munoz J, et al. Immunological properties of mesenchymal stem cells and clinical implications. Arch Immunol Ther Exp 2008;56:1-8.

18 Mushahary D, Spittler A, Kasper C, et al. Isolation, cultivation, and characterization of human mesenchymal stem cells. Cytometry $A$ 2018;93:19-31.

19 in 't Anker PS, Noort WA, Scherjon SA, et al. Mesenchymal stem cells in human second-trimester bone marrow, liver, lung, and spleen exhibit a similar immunophenotype but a heterogeneous multilineage differentiation potential. Haematologica 2003;88:845-52.

20 Ilancheran S, Moodley Y, Manuelpillai U. Human fetal membranes: a source of stem cells for tissue regeneration and repair? Placenta 2009;30:2-10.

21 Götherström C, Ringdén O, Tammik C, et al. Immunologic properties of human fetal mesenchymal stem cells. Am J Obstet Gynecol 2004;190:239-45.

22 Campagnoli C, Roberts IA, Kumar S, et al. Identification of mesenchymal stem/progenitor cells in human first-trimester fetal blood, liver, and bone marrow. Blood 2001;98:2396-402.

23 Ho AD, Wagner W, Franke W. Heterogeneity of mesenchymal stroma cell preparations. Cytotherapy 2008;10:320-30.

24 Dominici M, Le Blanc K, Mueller I, et al. Minimal criteria for defining multipotent mesenchymal stromal cells. The International Society for cellular therapy position statement. Cytotherapy 2006;8:315-7.

25 Krampera M, Galipeau J, Shi Y, et al. Immunological characterization of multipotent mesenchymal stromal cells - The International Society for Cellular Therapy (ISCT) working proposal. Cytotherapy 2013;15:1054-61.

26 Ankrum JA, Ong JF, Karp JM. Mesenchymal stem cells: immune evasive, not immune privileged. Nat Biotechnol 2014;32:252-60.

27 Song N, Scholtemeijer M, Shah K. Mesenchymal stem cell immunomodulation: mechanisms and therapeutic potential. Trends Pharmacol Sci 2020;41:653-64.

28 Weiss ARR, Dahlke MH. Immunomodulation by mesenchymal stem cells (MscS): mechanisms of action of living, apoptotic, and dead MscS. Front Immunol 2019:10:1191.

29 Spaeth E, Klopp A, Dembinski J, et al. Inflammation and tumor microenvironments: defining the migratory itinerary of mesenchymal stem cells. Gene Ther 2008;15:730-8.

30 Marofi F, Vahedi G, Biglari A, et al. Mesenchymal stromal/stem cells: a new era in the cell-based targeted gene therapy of cancer. Front Immunol 2017;8:8.

31 Choi S, Levy O, Coelho MB, et al. A cell rolling cytometer reveals the correlation between mesenchymal stem cell dynamic adhesion and differentiation state. Lab Chip 2014;14:161-6.

32 Reagan MR, Kaplan DL. Concise review: mesenchymal stem cell tumor-homing: detection methods in disease model systems. Stem Cells 2011;29:920-7.

33 Lin W, Huang L, Li Y, et al. Mesenchymal stem cells and cancer: clinical challenges and opportunities. Biomed Res Int 2019;2019:1-12.

34 Foster DS, Jones RE, Ransom RC, et al. The evolving relationship of wound healing and tumor stroma. JCl Insight 2018;3:e99911.

35 Sundaram GM, Quah S, Sampath P. Cancer: the dark side of wound healing. Febs $J$ 2018;285:4516-34. 
36 Li P, Gong Z, Shultz LD, et al. Mesenchymal stem cells: from regeneration to cancer. Pharmacol Ther 2019;200:42-54.

37 Galland S, Stamenkovic I. Mesenchymal stromal cells in cancer: a review of their immunomodulatory functions and dual effects on tumor progression. J Pathol 2020;250:555-72.

38 Manferdini C, Paolella F, Gabusi E, et al. Adipose stromal cells mediated switching of the pro-inflammatory profile of M1-like macrophages is facilitated by PGE2: in vitro evaluation. Osteoarthritis Cartilage 2017;25:1161-71

39 Lo Sicco C, Reverberi D, Balbi C, et al. Mesenchymal stem cellderived extracellular vesicles as mediators of anti-inflammatory effects: endorsement of macrophage polarization: MSC-derived EVs promote macrophage polarization. STEM CELLS Trans/ Med 2017;6:1018-28.

40 Chen $\mathrm{H}-\mathrm{W}$, Chen $\mathrm{H}-\mathrm{Y}$, Wang L-T, et al. Mesenchymal stem cells tune the development of monocyte-derived dendritic cells toward a myeloid-derived suppressive phenotype through growth-regulated oncogene chemokines. J Immunol 2013;190:5065-77.

41 Reis M, Mavin E, Nicholson L, et al. Mesenchymal stromal cellderived extracellular vesicles attenuate dendritic cell maturation and function. Front Immunol 2018;9:2538.

42 Galland S, Vuille J, Martin P, et al. Tumor-Derived mesenchymal stem cells use distinct mechanisms to block the activity of natural killer cell subsets. Cell Rep 2017;20:2891-905.

43 Batlle E, Massagué J. Transforming growth factor- $\beta$ signaling in immunity and cancer. Immunity 2019;50:924-40.

44 Martinet L, Fleury-Cappellesso S, Gadelorge M, et al. A regulatory cross-talk between $\mathrm{V} \gamma 9 \mathrm{~V} \delta 2 \mathrm{~T}$ lymphocytes and mesenchymal stem cells: immunomodulation. Eur J Immunol 2009;39:752-62.

45 Poggi A, Musso A, Dapino I, et al. Mechanisms of tumor escape from immune system: role of mesenchymal stromal cells. Immunol Lett 2014;159:55-72.

46 Ren G, Zhao X, Zhang L, et al. Inflammatory cytokineinduced intercellular adhesion molecule-1 and vascular cell adhesion molecule-1 in mesenchymal stem cells are critical for immunosuppression. J Immunol 2010;184:2321-8.

47 Patel SA, Meyer JR, Greco SJ, et al. Mesenchymal stem cells protect breast cancer cells through regulatory T cells: role of mesenchymal stem cell-derived TGF-beta. J Immunol 2010;184:5885-94.

48 Mohr A, Zwacka R. The future of mesenchymal stem cell-based therapeutic approaches for cancer - From cells to ghosts. Cancer Lett 2018;414:239-49.

49 Josiah DT, Zhu D, Dreher F, et al. Adipose-Derived stem cells as therapeutic delivery vehicles of an oncolytic virus for glioblastoma. Mol Ther 2010;18:377-85.

50 Jazowiecka-Rakus J, Sochanik A, Rusin A, et al. Myxoma VirusLoaded mesenchymal stem cells in experimental oncolytic therapy of murine pulmonary melanoma. Molecular Therapy - Oncolytics 2020;18:335-50.

51 Leoni V, Gatta V, Palladini A, et al. Systemic delivery of HER2retargeted oncolytic-HSV by mesenchymal stromal cells protects from lung and brain metastases. Oncotarget 2015;6:34774-87.

52 Du W, Seah I, Bougazzoul O, et al. Stem cell-released oncolytic herpes simplex virus has therapeutic efficacy in brain metastatic melanomas. Proc Natl Acad Sci U S A 2017:114:E6157-65.

53 Ong H-T, Federspiel MJ, Guo CM, et al. Systemically delivered measles virus-infected mesenchymal stem cells can evade host immunity to inhibit liver cancer growth. J Hepatol 2013;59:999-1006.

54 Castleton A, Dey A, Beaton B, et al. Human mesenchymal stromal cells deliver systemic oncolytic measles virus to treat acute lymphoblastic leukemia in the presence of humoral immunity. Blood 2014;123:1327-35

55 Mader EK, Maeyama Y, Lin Y, et al. Mesenchymal stem cell carriers protect oncolytic measles viruses from antibody neutralization in an orthotopic ovarian cancer therapy model. Clin Cancer Res 2009; $15: 7246-55$

56 Mader EK, Butler G, Dowdy SC, et al. Optimizing patient derived mesenchymal stem cells as virus carriers for a phase I clinical trial in ovarian cancer. J Trans/ Med 2013;11:20.

57 Hakkarainen T, Särkioja M, Lehenkari P, et al. Human mesenchymal stem cells lack tumor tropism but enhance the antitumor activity of oncolytic adenoviruses in orthotopic lung and breast tumors. Hum Gene Ther 2007;18:627-41.

58 Stoff-Khalili MA, Rivera AA, Mathis JM, et al. Mesenchymal stem cells as a vehicle for targeted delivery of CRAds to lung metastases of breast carcinoma. Breast Cancer Res Treat 2007;105:157-67.

59 Yong RL, Shinojima N, Fueyo J, et al. Human bone marrowderived mesenchymal stem cells for intravascular delivery of oncolytic adenovirus Delta24-RGD to human gliomas. Cancer Res 2009:69:8932-40.
60 Shinojima N, Hossain A, Takezaki T, et al. TGF- $\beta$ mediates homing of bone marrow-derived human mesenchymal stem cells to glioma stem cells. Cancer Res 2013;73:2333-44.

61 Bolontrade MF, Sganga L, Piaggio E, et al. A specific subpopulation of mesenchymal stromal cell carriers overrides melanoma resistance to an oncolytic adenovirus. Stem Cells Dev 2012;21:2689-702.

62 Huang Y-F, Chen M-J, Wu M-H, et al. The use of hypoxic cultured mesenchymal stem cell for oncolytic virus therapy. Cancer Gene Ther 2013:20:308-16

63 Li Z, Ye Z, Zhang X, et al. E1A-engineered human umbilical cord mesenchymal stem cells as carriers and amplifiers for adenovirus suppress hepatocarcinoma in mice. Oncotarget 2016;7:51815-28.

64 Yuan X, Zhang Q, Li Z, et al. Mesenchymal stem cells deliver and release conditionally replicative adenovirus depending on hepatic differentiation to eliminate hepatocellular carcinoma cells specifically. Cancer Lett 2016;381:85-95.

65 Yoon A-R, Hong J, Li Y, et al. Mesenchymal stem cell-mediated delivery of an oncolytic adenovirus enhances antitumor efficacy in hepatocellular carcinoma. Cancer Res 2019;79:4503-14.

66 Mahasa KJ, de Pillis L, Ouifki R, et al. Mesenchymal stem cells used as carrier cells of oncolytic adenovirus results in enhanced oncolytic virotherapy. Sci Rep 2020;10:425.

$67 \mathrm{Na} \mathrm{Y,} \mathrm{Nam} \mathrm{J-P,} \mathrm{Hong} \mathrm{J,} \mathrm{et} \mathrm{al.} \mathrm{Systemic} \mathrm{administration} \mathrm{of} \mathrm{human}$ mesenchymal stromal cells infected with polymer-coated oncolytic adenovirus induces efficient pancreatic tumor homing and infiltration. $J$ Control Release 2019;305:75-88.

68 Kaczorowski A, Hammer K, Liu L, et al. Delivery of improved oncolytic adenoviruses by mesenchymal stromal cells for elimination of tumorigenic pancreatic cancer cells. Oncotarget 2016;7:9046-59.

69 Guo Y, Zhang Z, Xu X, et al. Menstrual blood-derived stem cells as delivery vehicles for oncolytic adenovirus virotherapy for colorectal cancer. Stem Cells Dev 2019;28:882-96.

70 Hoyos V, Del Bufalo F, Yagyu S, et al. Mesenchymal stromal cells for linked delivery of oncolytic and apoptotic adenoviruses to non-smallcell lung cancers. Mol Ther 2015;23:1497-506.

71 Moreno R, Rojas LA, Villellas FV, et al. Human menstrual bloodderived mesenchymal stem cells as potential cell carriers for oncolytic adenovirus. Stem Cells Int 2017;2017:1-10.

72 Moreno R, Fajardo CA, Farrera-Sal M, et al. Enhanced antitumor efficacy of oncolytic Adenovirus-loaded menstrual blood-derived mesenchymal stem cells in combination with peripheral blood mononuclear cells. Mol Cancer Ther 2019;18:127-38.

73 Barlabé P, Sostoa Jde, Fajardo CA, et al. Enhanced antitumor efficacy of an oncolytic adenovirus armed with an EGFR-targeted bite using menstrual blood-derived mesenchymal stem cells as carriers. Cancer Gene Ther 2020;27:383-8.

74 Martinez-Quintanilla J, He D, Wakimoto $\mathrm{H}$, et al. Encapsulated stem cells loaded with hyaluronidase-expressing oncolytic virus for brain tumor therapy. Mol Ther 2015;23:108-18

75 Ryu W-S. Virus life cycle. Mol Virol Hum Pathog Viruses 2017:31-45.

76 Cascallo M, Alonso MM, Rojas JJ, et al. Systemic toxicity-efficacy profile of ICOVIR-5, a potent and selective oncolytic adenovirus based on the pRB pathway. Mol Ther 2007;15:1607-15.

77 García-Castro J, Alemany R, Cascalló M, et al. Treatment of metastatic neuroblastoma with systemic oncolytic virotherapy delivered by autologous mesenchymal stem cells: an exploratory study. Cancer Gene Ther 2010;17:476-83.

78 Melen GJ, Franco-Luzón L, Ruano D, et al. Influence of carrier cells on the clinical outcome of children with neuroblastoma treated with high dose of oncolytic adenovirus delivered in mesenchymal stem cells. Cancer Lett 2016;371:161-70.

79 Ruano D, López-Martín JA, Moreno L, et al. First-In-Human, First-inChild trial of autologous MscS carrying the oncolytic virus Icovir-5 in patients with advanced tumors. Mol Ther 2020;28:1033-42.

80 Bunnell BA, Betancourt AM, Sullivan DE. New concepts on the immune modulation mediated by mesenchymal stem cells. Stem Cell Res Ther 2010;1:34.

81 Waterman RS, Tomchuck SL, Henkle SL, et al. A new mesenchymal stem cell (MSC) paradigm: polarization into a pro-inflammatory Msc1 or an immunosuppressive MSC2 phenotype. PLoS One 2010;5:e10088

82 Waterman RS, Henkle SL, Betancourt AM. Mesenchymal stem cell 1 (MSC1)-based therapy attenuates tumor growth whereas MSC2-treatment promotes tumor growth and metastasis. PLoS One 2012;7:e45590.

83 Galland S, Stamenkovic I. Mesenchymal stromal cells in cancer: a review of their immunomodulatory functions and dual effects on tumor progression. J Pathol 2020;250:555-72. 
Correction: Mesenchymal stem cells and oncolytic viruses: joining forces against cancer

Moreno R. Mesenchymal stem cells and oncolytic viruses: joining forces against cancer. J Immunother Cancer 2021;9:e001684. doi: 10.1136/jitc-2020-001684

This paper has been updated since first published to add reference 49 .

Open access This is an open access article distributed in accordance with the Creative Commons Attribution Non Commercial (CC BY-NC 4.0) license, which permits others to distribute, remix, adapt, build upon this work non-commercially, and license their derivative works on different terms, provided the original work is properly cited, appropriate credit is given, any changes made indicated, and the use is non-commercial. See http://creativecommons.org/licenses/by-nc/4.0/.

(C) Author(s) (or their employer(s)) 2021. Re-use permitted under CC BY-NC. No commercial re-use. See rights and permissions. Published by BMJ.

J Immunother Cancer 2021;9:e001684corr1. doi:10.1136/jitc-2020-001684corr1

D Check for updates 